\title{
Circulating Tumor Cells - Cutting Edge Research?
}

\section{Yuen WM John ${ }^{1 *}$ and Gohel I Mayur-Danny ${ }^{2}$}

${ }^{1}$ School of Nursing, The Hong Kong Polytechnic University, Hung Hom, Kowloon, Hong Kong Special Administrative Region

${ }^{2}$ Department of Medical Science, Tung Wah College, Wylie Road, Homantin, Kowloon, Hong Kong Special Administrative Region

"Circulating Tumor Cells (CTCs)", shed into the bloodstream from primary and metastatic tumors are regarded as the liquid biopsy of cancer is an enthusiastic field of research which attracted half-athousand publications in the recent two decades. CTCs were first reported in the blood of a dead cancer patient in 1869 [1]. The CTCs research can be categorized into three major aspects: 1) Clinically focused on the applications of CTCs as biomarkers for cancer diagnosis, progression, prognosis and therapeutics; 2) Molecular characterization of CTCs to determine their clinical relevance and functional activities in tumor microenvironments; and 3) Technological investment in detection and isolation of rare CTCs from peripheral blood specimens and even in vivo tracking of CTCs.

The current chemistry of CTC analysis is, as applied by the only FDA-cleared CellSearch ${ }^{\circledR}$ system and other platforms, mainly relied on differentiating live epithelial cells from peripheral CD45+ leukocytes, by using epithelial makers, such as EpCAM, CK 8, 18 and 19 in addition to viability DAPI marker [2]. Based on this principle, high CTC numbers have been correlated with increased metastasis and decreased time to relapse, which supporting the predictive values on cancer progression, prognosis and survival in several cancers [3]. A meta-analysis of 49 eligible studies enrolling 6,825 breast cancer patients indicated CTC a stable prognostic biomarker at both earlyand metastatic stages [4]. Such encouraging reports have directed many investigators into the search of various strategies in CTC enrichment to achieve the limit of detection at one CTC per million cells level. According to Hong and $\mathrm{Zu}$ [5], from the year 2004 to 2012, at least 14 microdevice platforms have been developed following the CellSearch ${ }^{\circledR}$, and they have competed with each other for the clinical sensitivity, specificity, viability, recovery and purity. Some investigators even intended to develop a single platform that is able to detect all cancers [6]. However, the approach of using epithelial markers may cause false positives such as wrong identification of benign circulating epithelial cells, otherwise more importantly omitting the biological features of CTCs such as heterogeneity, subpopulational stemness, partial epithelial-mesenchymal transition, and seeding potential [7]. AlixPanabieres and Pantel [2] has emphasized the important interplays between Epithelial-Mesenchymal Transition (EMT) and MesenchymalEpithelial Transition (MET) in cancer disease, which may influence the moiety of molecular expression in relation to dedifferentiation property and cell plasticity. Have the CTC research gone too fast? What are the real challenges? Several important hurdles about CTCs remain unsolved. One is the biophysical factors such as trapping at the capillary pores that leads to only a minute amount and/or plastic CTCs to be present in the circulation [7]. This at least partially explains why application varies due to the tumor origin, for example CTCs can be detected in early-staged breast tumors but cannot in colorectal tumors. In patients with colorectal cancer, the CTCs recovery was shown to be more efficient in mesenteric blood than peripheral blood, suggesting that CTCs as biomarkers may require more invasive ways rather than the minimally invasive venipuncture depending on the tumor site [8]. In order to further break through the CTC enrichment and its detection, researchers may have to go a bit back to the basic science, i.e. the characterization of the real CTCs rather than the "circulating epithelial cells". This would require the help of cutting-edge technology for viable CTC isolation that allow the culture and analysis of primary cancer cells [9]. Last but not the least, an in vivo enrichment in the arm vein of patients has been described and shown to enrich 10 times the sensitivity for CTC detection [10]. This has primed the idea of real- time in vivo tracking of CTCs by imaging techniques and also possibly therapeutic targets, in near future, especially when the specific fingerprints of CTCs are decoded.

\section{References}

1. Asworth TR (1869) A case of cancer in which cells similar to those in tumors were seen in the blood after death. Australian Medical Journal 14: 146-149.

2. Alix-Panabières C, Pantel K (2013) Circulating tumor cells: liquid biopsy of cancer. Clin Chem 59: 110-118.

3. Balic M, Williams A, Lin H, Datar R, Cote RJ (2013) Circulating tumor cells: from bench to bedside. Annu Rev Med 64: 31-44.

4. Zhang L, Riethdorf S, Wu G, Wang T, Yang K, et al. (2012) Meta-analysis of the prognostic value of circulating tumor cells in breast cancer. Clin Cancer Res 18: $5701-5710$.

5. Hong B, Zu Y (2013) Detecting circulating tumor cells: current challenges and new trends. Theranostics 3: 377-394.

6. Ozkumur E, Shah AM, Ciciliano JC, Emmink BL, Miyamoto DT, et al. (2013) Inertial focusing for tumor antigen-dependent and independent sorting of rare circulating tumor cells. Sci Transl Med 5: 179ra47.

7. Plaks V, Koopman CD, Werb Z (2013) Circulating tumor cells. Science 341 1186-1188.

8. Deneve E, Riethdorf S, Ramos J, Nocca D, Coffy A, et al. (2013) Capture of viable circulating tumor cells in the liver of colorectal cancer patients. Clin Chem 59: 1384-1392.

9. Hughes AD, Mattison J, Powderly JD, Greene BT, King MR (2012) Rapid isolation of viable circulating tumor cells from patient blood samples. J Vis Exp : e4248.

10. Saucedo-Zeni N, Mewes S, Niestroj R, Gasiorowski L, Murawa D, et al. (2012) A novel method for the in vivo isolation of circulating tumor cells from peripheral blood of cancer patients using a functionalized and structured medical wire. Int J Oncol 41: 1241-1250.

*Corresponding author: John WM Yuen, School of Nursing, the Hong Kong Polytechnic University, Yuk Choi Road, Hung Hom, Kowloon, Hong Kong Special Administrative Region, Tel: +852-2766-4130; Fax: +852-2364-9663; E-mail: john.yuen@polyu.edu.hk

Received November 24, 2013; Accepted November 26, 2013; Published December 02, 2013

Citation: John YWM, Mayur-Danny GI (2013) Circulating Tumor Cells - Cutting Edge Research? Mod Chem appl 1: e117. doi:10.4172/2329-6798.1000e117

Copyright: (c) 2013 John YWM, et al. This is an open-access article distributed under the terms of the Creative Commons Attribution License, which permits unrestricted use, distribution, and reproduction in any medium, provided the original author and source are credited. 